\title{
Analysis of normalized point source sensitivity as a performance metric for large telescopes
}

\author{
Byoung-Joon Seo, ${ }^{1,2, \star}$ Carl Nissly, ${ }^{1,2}$ George Angeli, ${ }^{3}$ Brent Ellerbroek, ${ }^{3}$ \\ Jerry Nelson, ${ }^{3}$ Norbert Sigrist, ${ }^{1,2}$ and Mitchell Troy ${ }^{1,2}$ \\ 1Jet Propulsion Laboratory, Pasadena, California, 91109, USA \\ ${ }^{2}$ California Institute of Technology, Pasadena, California, 91125, USA \\ ${ }^{3}$ Thirty Meter Telescope Observatory, Pasadena, California, 91107, USA \\ ${ }^{*}$ Corresponding author: Byoung-Joon.Seo@jpl.nasa.gov
}

Received 1 June 2009; revised 28 September 2009; accepted 30 September 2009; posted 7 October 2009 (Doc. ID 112048); published 27 October 2009

\begin{abstract}
We investigate a new metric, the normalized point source sensitivity (PSSN), for characterizing the seeing-limited performance of large telescopes. As the PSSN metric is directly related to the photometric error of background limited observations, it represents the efficiency loss in telescope observing time. The PSSN metric properly accounts for the optical consequences of wave front spatial frequency distributions due to different error sources, which differentiates from traditional metrics such as the $80 \%$ encircled energy diameter and the central intensity ratio. We analytically show that multiplication of individual PSSN values due to individual errors is a good approximation for the total PSSN when various errors are considered simultaneously. We also numerically confirm this feature for Zernike aberrations as well as for the numerous error sources considered in the error budget of the Thirty Meter Telescope (TMT) using a ray optics simulator. Additionally, we discuss other pertinent features of the PSSN, including its relations to Zernike aberration, RMS wave front error, and central intensity ratio. () 2009 Optical Society of America

OCIS codes: $110.6770,110.3925$.
\end{abstract}

\section{Introduction}

The optical performance of an extremely large astronomical telescope, such as the Thirty Meter Telescope (TMT), is a major concern for the astronomers and engineers developing these next generation, ground based facilities $[\underline{1}, 2]$. While the design should be guided by the optimization of this performance, performance degradation should also be allocated among the various components of the observatory, i.e., an error budget should be established. "Performance" being an abstract term, these operations can only be carried out through an appropriate performance metric. First and foremost, an adequate performance metric should reflect the scientific capabilities of the observatory. In addition, it

0003-6935/09/315997-11\$15.00/0

(C) 2009 Optical Society of America is desirable to predict or estimate overall performance of the telescope when aberrations from multiple error sources are combined. This estimation feature of a metric is especially important when designing and budgeting errors for a large telescope such as TMT, since its design and modeling tasks are highly distributed to multiple optical designers, vendors, and manufacturers [3].

The root mean square of the wave front error (RMS WFE), 80\% encircled energy diameter (EE80), and central intensity ratio (CIR) are often used as performance metrics for astronomical telescopes. In the case of small and mostly random (uncorrelated) aberrations, like the residuals after adaptive optics (AO) corrections, the RMS WFE is the universally accepted metric. Multiple, statistically independent effects can be combined in an error budget by adding the wave front variances of the individual aberrations, that is, by calculating the root sum square 
(RSS) of the RMS WFEs. However, if the aberrations are relatively large, as during seeing-limited observation without $\mathrm{AO}$ corrections, the RMS WFE fails in describing the telescope performance well enough. It is so because higher spatial frequency aberrations degrade many types of science more than lower frequency aberrations, even if they both have the same RMS WFE. The CIR [4] was developed to mitigate the problems associated with the larger optical errors of seeing-limited observations. However, as an extension to the Strehl ratio, it considers only the central intensity of the point spread function (PSF); therefore it is also insufficient for describing spatial contributions of telescope aberrations. On the other hand, while the EE80 metric is known to describe science performance well, overall assessment from multiple error sources (error budgeting) is challenging since this metric has nonlinear characteristics when the errors are within the physical optics regime.

We consequently propose a new metric, the normalized point source sensitivity (PSSN), for characterizing the seeing-limited performance of large telescopes. This metric is directly related to the photometric error of background limited observations so that it accurately represents the efficiency loss of science due to increased telescope observing time. The PSSN metric also properly accounts for the consequences of different spatial frequency distributions for different wave front error sources, which makes it superior to traditional metrics such as CIR and EE80 for estimating seeing-limited errors. The combination of contributions from multiple error sources is also well understood.

This paper is organized as follows: In Section 2, we first introduce the definition of the PSSN along with its physical interpretation. After we summarize the properties of the PSSN in Section $\underline{3}$, we verify these properties analytically and numerically in Sections 4 and $\underline{5}$, respectively. We conclude in Section $\underline{6}$.

\section{Definition}

As King [5] pointed out, the photometric error, i.e., the intensity variance of an astronomical point source (star) due to sky background depends on the star intensity $I_{\text {star }}$, the background irradiance $b$, and the shape of the PSF of the optics. For background limited observations $\left(b \gg I_{\text {star }}\right)$, this photometric error $\sigma_{\text {int }}^{2}$ is proportional to the background and inversely proportional to the integral square of the PSF:

$$
\sigma_{\text {int }}^{2}=\frac{I_{\text {star }}}{\int_{\infty} \frac{|\operatorname{PSF}(\vec{\theta})|^{2}}{\operatorname{PSF}(\vec{\theta})+b / I_{\text {star }}} \mathrm{d} \vec{\theta}} \approx \frac{b}{\int_{\infty}|\operatorname{PSF}(\vec{\theta})|^{2} \mathrm{~d} \vec{\theta}},
$$

where $\vec{\theta}$ is the two-dimensional coordinate for the $\mathrm{PSF}$, typically in units of radians (or meter). We will call the integral square of the PSF the point source sensitivity (PSS) of the telescope:

$$
\operatorname{PSS} \equiv \int_{\infty}|\operatorname{PSF}(\vec{\theta})|^{2} \mathrm{~d} \vec{\theta}
$$

The signal-to-noise ratio (SNR) of a background limited astronomical observation can be improved by increasing the integration time $T$ :

$$
\mathrm{SNR}=\frac{I_{\mathrm{star}} T}{\sqrt{\sigma_{\mathrm{int}}^{2} T}} \approx \sqrt{\frac{\mathrm{PSS}}{b}} \sqrt{T} .
$$

Assuming the same target SNR, the relative integration time increase due to telescope aberrations depends only on the corresponding change in PSS. By introducing the normalized point source sensitivity (PSSN), we now have a metric directly related to the scientific productivity of the telescope:

$$
\operatorname{PSSN}=\frac{\int_{\infty}\left|\operatorname{PSF}_{t+a+e}(\vec{\theta})\right|^{2} \mathrm{~d} \vec{\theta}}{\int_{\infty}\left|\operatorname{PSF}_{t+a}(\vec{\theta})\right|^{2} \mathrm{~d} \vec{\theta}},
$$

where $\mathrm{PSF}_{t+a}$ is the time-averaged PSF of the ideal telescope exposed to the atmosphere and $\mathrm{PSF}_{t+a+e}$ is the time-averaged PSF of an aberrated telescope exposed to the atmosphere. The subscripts $t, a$, and $e$ in $\mathrm{PSF}_{t+a}$ and $\mathrm{PSF}_{t+a+e}$ represent that the optical transfer function (OTF) is modified due to the telescope aperture, atmosphere, and telescope error, respectively. We will use this notation throughout this paper.

A different line of thought, based on linear system theory, leads us to the same metric. Figure 1 shows the conceptual schematic of an optical system (such as an astronomical telescope), where the system input and output are defined as the spatial intensity spectra $I_{g}$ and $I_{i}$ of the perfect (geometric) and aberrated images, respectively. The optical system is characterized by its OTF, linking the output to the input in a linear way. The source of the perturbation is denoted as the plant $P$, which includes both atmospheric and telescope aberration. The perturbations are corrected by active and adaptive optics systems indicated by the $K$ feedback loop. The objective in designing and controlling an optical system is

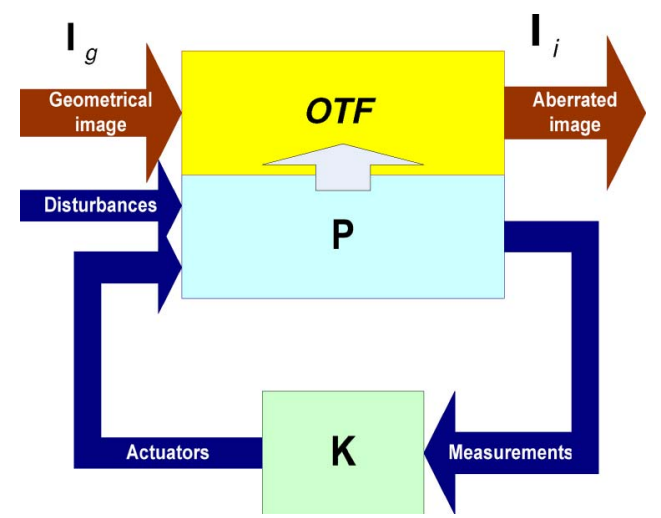

Fig. 1. (Color online) Conceptual schematic for an optical system. 
"maximizing" $I_{i}$ for all potential $I_{g}$; that is, "maximizing" the system. While there are various ways to define the "size" of a linear system; the most common and generally useful way to do so is by its $H_{2}$ norm, based on the integral square of the OTF.

Since the OTF and the PSF are related through the Fourier transformation according to the Parseval's energy conservation theorem, the $H_{2}$ norm can also be represented using the PSF:

$$
H_{2}^{2}=\int_{\infty}|\mathrm{OTF}(\vec{f})|^{2} \mathrm{~d} \vec{f}=\int_{\infty}|\operatorname{PSF}(\vec{\theta})|^{2} \mathrm{~d} \vec{\theta}=\mathrm{PSS},
$$

where $\vec{f}$ is the two-dimensional coordinate for the OTF, typically in units of cycles per radian (or cycles per meter). Therefore, the proposed metric, PSSN, can also be expressed as

$$
\operatorname{PSSN}=\frac{\int_{\infty}\left|\mathrm{OTF}_{t+a+e}(\vec{f})\right|^{2} \mathrm{~d} \vec{f}}{\int_{\infty}\left|\mathrm{OTF}_{t+a}(\vec{f})\right|^{2} \mathrm{~d} \vec{f}},
$$

where $\mathrm{OTF}_{t+a}$ and $\mathrm{OTF}_{t+a+e}$ are the OTFs of $\mathrm{PSF}_{t+a}$ and $\mathrm{PSF}_{t+a+e}$, respectively.

It is worth noting that for diffraction limited observations by space telescopes or adaptive optics aided ground based observatories the relevant metric is PSS, as the reference of course is the perfect image. It was suggested by Muller [6] and later investigated by Christou [7] as the $S 1$ metric of sharpness.

\section{Properties}

Based on its definition, we find that the PSSN has the following properties, discussed in the remainder of this paper.

$\boldsymbol{P 1}$ The PSSN becomes unity when there are no telescope errors and 0 when the error is infinitely large:

$$
0 \leq \mathrm{PSSN} \leq 1 .
$$

P2 The PSSN has a multiplicative feature for low frequency and weak aberrations. Suppose there are multiple errors on the telescope. Let $\operatorname{PSSN}_{C}$ and $\mathrm{PSSN}_{M}$ be the PSSN for all combined error and for multiplied values of PSSN from individual errors, respectively. Then, $\mathrm{PSSN}_{M}$ approximates $\operatorname{PSSN}_{C}$ :

$$
\operatorname{PSSN}_{C} \approx \mathrm{PSSN}_{M} .
$$

P3 The PSSN has a relation to RMS WFE if we assume the RMS WFE is small:

$$
\mathrm{PSSN} \approx 1-\alpha \sigma^{2},
$$

where $\sigma$ is the RMS WFE and $\alpha$ is a proportional constant independent to $\sigma$. However, $\alpha$ is a function of wavelength, aberration frequency [correlation length of the optical path difference (OPD)], and Fried parameter, $r_{o}$.
$\boldsymbol{P} 4$ The PSSN can be approximated to the square of the Strehl ratio for white noise aberration ( 0 correlation length of OPD). This is because the energy in PSF spreads out beyond the Fried parameter, $r_{o}$. Therefore,

$$
\mathrm{PSSN} \approx 1-2 \sigma^{2}
$$

where $\sigma$ is the RMS WFE in radians and $\alpha$ becomes $2 / \mathrm{rad}^{2}$

$\boldsymbol{P} 5$ The PSSN has a relation to the central intensity ratio (CIR) [4] as

$$
\frac{1-\mathrm{PSSN}}{1-\mathrm{CIR}} \approx \gamma,
$$

where $\gamma$ is a proportional constant and a function of wavelength, aberration frequency, and atmosphere $r_{o}$. The constant $\gamma$ becomes 2 for white noise aberrations since the CIR becomes the Strehl ratio for white noise.

\section{Analytical Analysis}

\section{A. Simplification of Normalized Point Source Sensitivity}

The PSF (before exposed to atmosphere) due to an aberrated telescope can be represented as

$$
\operatorname{PSF}_{t+e}(\vec{\theta})=\left|\int_{r} A(\vec{r}) e^{-j k \mathrm{OPD}(\vec{r})} e^{j \vec{\theta} \vec{r}} \mathrm{~d} \vec{r}\right|^{2},
$$

where $\vec{r}$ and $\mathrm{d} \vec{r}$ are the two-dimensional coordinates for the OPD (typically in meter units); $k$ is the wave number defined as $2 \pi / \lambda ; \lambda$ is the wavelength; and $A(\vec{r})$ is the real, nonnegative telescope pupil function normalized with $\int A^{2}(\vec{r}) \mathrm{d} \vec{r}=1$. Using the Fourier transformation properties and integration properties, the OTF of $\mathrm{PSF}_{t+e}$ is obtained as

$$
\mathrm{OTF}_{t+e}(\vec{f})=\int_{r} A(\vec{r}) A(\vec{r}-\lambda \vec{f}) e^{-j \Omega(\vec{r}, \lambda \vec{f})} \mathrm{d} \vec{r},
$$

where $\Omega(\vec{r}, \lambda \vec{f})$ is defined as the OPD difference between $\vec{r}$ and $\vec{r}-\lambda \vec{f}$ in radians,

$$
\Omega(\vec{r}, \lambda \vec{f}) \equiv k(\operatorname{OPD}(\vec{r})-\operatorname{OPD}(\vec{r}-\lambda \vec{f})) .
$$

We will use $\Omega$ for representing $\Omega(\vec{r}, \lambda \vec{f})$ in the remainder of this paper for simplicity. We obtain the $\mathrm{OTF}_{t+a+e}$, time-averaged OTF of the aberrated telescope exposed to the atmosphere if we consider the exposed OTF of the atmosphere,

$$
\mathrm{OTF}_{t+a+e}(\vec{f})=\mathrm{OTF}_{a}(\vec{f}) \cdot \int_{r} A(\vec{r}) A(\vec{r}-\lambda \vec{f}) e^{-j \Omega} \mathrm{d} \vec{r},
$$

where $\mathrm{OTF}_{a}(\vec{f})$ is the time-averaged OTF of the atmosphere. 
We define $\mathrm{OTF}_{e}$ such that

$$
\mathrm{OTF}_{e}(\vec{f}) \equiv\left\langle e^{-j \Omega}\right\rangle_{r},
$$

where $\langle\cdot\rangle_{r}$ defines a new operator, average of overlapping pupil, such that

$$
\langle\cdot\rangle_{r} \equiv \frac{\int_{r} A(\vec{r}) A(\vec{r}-\lambda \vec{f})(\cdot) \mathrm{d} \vec{r}}{\int_{r} A(\vec{r}) A(\vec{r}-\lambda \vec{f}) \mathrm{d} \vec{r}} .
$$

Then the $\mathrm{OTF}_{t+a+e}$ can be represented as a multiplication of $\mathrm{OTF}_{t+a}$ and $\mathrm{OTF}_{e}$, where $\mathrm{OTF}_{t+a}$ is the time-averaged OTF of the ideal telescope exposed to the atmosphere, and $\mathrm{OTF}_{e}$ is the time-averaged OTF due to the telescope aberrations only; i.e.,

$$
\mathrm{OTF}_{t+a+e}(\vec{f})=\mathrm{OTF}_{t+a}(\vec{f}) \cdot \mathrm{OTF}_{e}(\vec{f})
$$

Using Eqs. (ㅁ) and (18), we simplify the definition of the PSSN as

$$
\mathrm{PSSN}=\left\langle\left|\mathrm{OTF}_{e}(\vec{f})\right|^{2}\right\rangle,
$$

where $\langle\cdot\rangle$ defines another new operator, average over the atmosphere, such that

$$
\langle\cdot\rangle \equiv \frac{\int_{\infty}(\cdot)\left|\mathrm{OTF}_{t+a}(\vec{f})\right|^{2} \mathrm{~d} \vec{f}}{\int_{\infty}\left|\mathrm{OTF}_{t+a}(\vec{f})\right|^{2} \mathrm{~d} \vec{f}} .
$$

Note that this operator is linear (i.e., $\langle A+B\rangle=\langle A\rangle+\langle B\rangle)$ and mathematically similar in many ways to the average operator used in random variable theory with the $\left|\mathrm{OTF}_{t+a}\right|^{2}$ being a probability density function. Thus, mathematically, PSSN is the average of $\left|\mathrm{OTF}_{e}\right|^{2}$ over $\left|\mathrm{OTF}_{t+a}\right|^{2}$. Note also that $0 \leq \mathrm{PSSN} \leq 1$, since $0 \leq\left|\mathrm{OTF}_{e}\right| \leq 1$ in Eq. (16), which proves $P 1$.

\section{B. Multiplicative Features of Normalized Point Source Sensitivity}

For most errors on modern large telescopes, we observe that the $\left|\mathrm{OTF}_{e}(\vec{f})\right|$ is much wider than the $\left|\mathrm{OTF}_{t+a}(\vec{f})\right|$. This implies that $\left|\mathrm{OTF}_{e}(\vec{f})\right|$ changes only by a small amount over the region of interest where $\left|\mathrm{OTF}_{t+a}(\vec{f})\right|$ is defined meaningfully. Therefore, for calculation convenience, we define a small valued positive real function, $\epsilon(\vec{f})$, such that

$$
\left|\mathrm{OTF}_{e}(\vec{f})\right|^{2}=1-\epsilon(\vec{f})
$$

Suppose that there are two errors on the telescope, which generate their $\left|\mathrm{OTF}_{e}(\vec{f})\right|^{2}$ values as $1-\epsilon_{1}(\vec{f})$ and $1-\epsilon_{2}(\vec{f})$. Then, the combined PSSN $\left(\mathrm{PSSN}_{C}\right)$ can be computed as

$$
\operatorname{PSSN}_{C}=1-\left\langle\epsilon_{1}(\vec{f})\right\rangle-\left\langle\epsilon_{2}(\vec{f})\right\rangle+\left\langle\epsilon_{m}(\vec{f})\right\rangle,
$$

where $\epsilon_{m}(\vec{f})$ is also a small valued function due to the cross talk between two aberrations. On the other hand, the multiplied PSSN ( $\operatorname{PSSN}_{M}$ ) from individual PSSNs can be denoted as

$$
\begin{aligned}
\operatorname{PSSN}_{M} & =\left\langle 1-\epsilon_{1}(\vec{f})\right\rangle\left\langle 1-\epsilon_{2}(\vec{f})\right\rangle \\
& =1-\left\langle\epsilon_{1}(\vec{f})\right\rangle-\left\langle\epsilon_{2}(\vec{f})\right\rangle+\left\langle\epsilon_{1}(\vec{f})\right\rangle\left\langle\epsilon_{2}(\vec{f})\right\rangle .
\end{aligned}
$$

The two equations in Eqs. (22) and (23) immediately imply that PSSN is multiplicative to the first order since the first order $\left\langle\epsilon_{i}\right\rangle$ values are small; therefore, their second orders are even smaller.

In order to further investigate the PSSN multiplicative feature beyond the first order, we first define the difference of Eqs. (22) and (23) as $\Delta$. Then,

$$
\Delta \equiv \operatorname{PSSN}_{C}-\operatorname{PSSN}_{M}=\left\langle\epsilon_{m}(\vec{f})\right\rangle-\left\langle\epsilon_{1}(\vec{f})\right\rangle\left\langle\epsilon_{1}(\vec{f})\right\rangle .
$$

We compute $\Delta$ analytically and decompose it into several terms using the Taylor expansion technique:

$$
\Delta=\Delta_{2}+\Delta_{4}+\Delta_{6}+\cdots,
$$

where $\Delta_{i}$ denotes the $i$ th order expansion term of $\Delta$. Note that odd orders vanish and the first and second dominant terms are the second and fourth orders, which can be calculated as

$$
\begin{aligned}
\Delta_{2}= & 2\left\langle\left\langle\Omega_{1}\right\rangle_{r}\left\langle\Omega_{2}\right\rangle_{r}-\left\langle\Omega_{1} \Omega_{2}\right\rangle_{r}\right\rangle \Delta_{4} \\
= & \left(\left\langle\left\langle\Omega_{1}^{2}\right\rangle_{r}\left\langle\Omega_{2}^{2}\right\rangle_{r}\right\rangle-\left\langle\left\langle\Omega_{1}^{2}\right\rangle_{r}\right\rangle\left\langle\left\langle\Omega_{2}^{2}\right\rangle_{r}\right\rangle\right) \\
& +\left(\left\langle\left\langle\Omega_{1}^{2}\right\rangle_{r}\left\langle\Omega_{1} \Omega_{2}\right\rangle_{r}\right\rangle+\left\langle\left\langle\Omega_{2}^{2}\right\rangle_{r}\left\langle\Omega_{1} \Omega_{2}\right\rangle_{r}\right\rangle\right. \\
& +\left\langle\left\langle\Omega_{1}^{2}\right\rangle_{r}\right\rangle\left\langle\left\langle\Omega_{2}\right\rangle_{r}^{2}\right\rangle+\left\langle\left\langle\Omega_{1}\right\rangle_{r}^{2}\right\rangle\left\langle\left\langle\Omega_{2}^{2}\right\rangle_{r}\right\rangle \\
& \left.-\left\langle\left\langle\Omega_{1}\right\rangle_{r}^{2}\right\rangle\left\langle\left\langle\Omega_{2}\right\rangle_{r}^{2}\right\rangle\right),
\end{aligned}
$$

where $\Omega_{1}$ and $\Omega_{2}$ are the corresponding $\Omega$ values in Eq. (14) for individual errors such that $\Omega=\Omega_{1}+\Omega_{2}$.

In order to further analyze the significance of $\Delta$, we make the following assumptions:

A1 Normalized power: We assume the total power on the aperture is unity. All OTFs have a value of one at the origin.

A2 Low order and weak aberration: As the error becomes smaller or the correlation length of OPD due to the error becomes larger than the atmosphere correlation length, $\mathrm{\epsilon}_{i}(\vec{f})$ becomes small relative to unity near the origin. This assumption results in the Taylor expansion approximation becoming valid for our analytical analysis. We consider $\Delta$ up to fourth order and $\epsilon_{i}(\vec{f})$ up to second order in terms of $\Omega_{i}$ as in Eq. (26).

A3 Independent aberrations: We assume multiple errors are statistically independent. By independent, we mean that two OPDs are uncorrelated over any region where the aperture is defined. Mathematically, we denote the dependence as 


$$
\begin{aligned}
& \int_{s} \int_{s^{\prime}} A(\vec{r}) A\left(\overrightarrow{r^{\prime}}\right) \mathrm{OPD}_{1}(\vec{r}) \mathrm{OPD}_{2}\left(\overrightarrow{r^{\prime}}\right) \mathrm{d} \vec{r} \mathrm{~d} \overrightarrow{r^{\prime}} \\
& =\int_{s} A(\vec{r}) \operatorname{OPD}_{1}(\vec{r}) \mathrm{d} \vec{r} \int_{s^{\prime}} A\left(\overrightarrow{r^{\prime}}\right) \mathrm{OPD}_{2}\left(\overrightarrow{r^{\prime}}\right) \mathrm{d} \overrightarrow{r^{\prime}},
\end{aligned}
$$

where $\mathrm{OPD}_{1}$ and $\mathrm{OPD}_{2}$ are two OPDs from two aberrations and are defined in $\vec{r}$ and $\overrightarrow{r^{\prime}}$ space, respectively. This assumption results in the following relation:

$$
\left\langle\Omega_{1} \Omega_{2}\right\rangle_{r}=\left\langle\Omega_{1}\right\rangle_{r}\left\langle\Omega_{2}\right\rangle_{r}
$$

Therefore, $\Delta_{2}$ vanishes in Eq. (26) and $\Delta_{4}$ becomes the most dominant term (i.e., $\Delta \approx \Delta_{4}$ ).

A4 Large and symmetric telescope: We assume that the diameter of the telescope is much larger than $\lambda \vec{f}$, where $\vec{f}$ is meaningfully defined due to the Fried parameter, $r_{0}$. We also assume that the telescope aperture is symmetric, i.e., $A(\vec{r})=A(-\vec{r})$. This assumption results in the following relation:

$$
\left\langle\Omega_{i}\right\rangle_{r} \approx 0 \text { for } i=1 \text { or } 2 .
$$

A5 Isotropic aberrations: We assume that the aberrations are isotropic; i.e., the $\mathrm{OTF}_{e}$ values are centro-symmetric in $f$ space and are represented as a function of $|\vec{f}|$. We also assume that $\mathrm{OTF}_{e}$ is approximated up to the second order with respect to $|\vec{f}|$.

A6 Long exposure atmosphere: The atmosphere OTF is described by the long exposure atmosphere [8], defined as

$$
\mathrm{OTF}_{a}(r)=\exp \left(-3.44\left(\frac{r}{r_{o} / \lambda}\right)^{5 / 3}\right)
$$

Suppose that assumptions in $A 1-A 4$ are valid; then one can find that $\Delta$ becomes simplified as follows:

$$
\Delta \approx\left\langle\epsilon_{1} \epsilon_{2}\right\rangle-\left\langle\epsilon_{1}\right\rangle\left\langle\epsilon_{2}\right\rangle,
$$

where we have dropped the symbol $\vec{f}$ in $\epsilon_{i}(\vec{f})$ for simplicity.

Note that $\Delta$ in Eq. (31) is mathematically similar to the statistic correlation in the random probability theory. According to the theory, $\Delta$ is bounded by the product of the standard deviations of $\epsilon_{1}(\vec{f})$ and $\epsilon_{2}(\vec{f})$,

$$
|\Delta| \leq \sqrt{\left(\left\langle\epsilon_{1}^{2}\right\rangle-\left\langle\epsilon_{1}\right\rangle^{2}\right)\left(\left\langle\epsilon_{2}^{2}\right\rangle-\left\langle\epsilon_{2}\right\rangle^{2}\right)},
$$

and becomes 0 when two $\epsilon_{i}(\vec{f})$ values are uncorrelated in $\vec{f}$ space.

Additional assumptions of $A 5$ and $A 6$ make $\Delta$ further simplified. Due to mathematical complexity, we detail the calculation in Appendix $\underline{\text { A: }}$

$$
\Delta \approx 1.2119\left(1-\mathrm{PSSN}_{1}\right)\left(1-\mathrm{PSSN}_{2}\right),
$$

where $\mathrm{PSSN}_{1}$ and $\mathrm{PSSN}_{2}$ are individual PSSNs of two error sources, and the constant 1.2119 repre- sents a dimensionless, unique value for the case of atmosphere we applied (long exposure atmosphere [8]). By using a Taylor series approximation up to the second order, note that $\Delta$ is a positive number and is equal to the maximum boundary in Eq. (32). Since we use the same positive polarity of coefficients for approximating $\epsilon_{1}(\vec{f})$ and $\epsilon_{2}(\vec{f})$, the result obtained in Eq. (33) represents the correlation coefficient of 1 ; thus, it is the same as the analytical boundary in Eq. (32). However, in general, $\Delta$ can be either positive or negative depending on the correlation between two $\epsilon_{i}(f)$ values. Therefore, Eq. (33) is reformulated using the analytical boundary $\Delta_{A}$ :

$$
|\Delta| \leq \Delta_{A} \approx 1.2119\left(1-\mathrm{PSSN}_{1}\right)\left(1-\mathrm{PSSN}_{2}\right) .
$$

Equation (34) implies that the difference $\Delta$ between $\mathrm{PSSN}_{C}$ and $\mathrm{PSSN}_{M}$ is within the analytical boundary $\Delta_{A}$; i.e,

$$
\left|\mathrm{PSSN}_{C}-\mathrm{PSSN}_{M}\right| \leq \Delta_{A}
$$

Note that $1-\mathrm{PSSN}_{i}$ is normally a small value; thus, $\Delta_{A}$ is on the order of the square of $1-\mathrm{PSSN}_{i}$. For example, let us assume that $\mathrm{PSSN}_{1}=\mathrm{PSSN}_{2}=0.95$. Then, $\Delta_{A}$ becomes $3 \times 10^{-3}$.

Furthermore, we consider $N$ different errors by generalizing Eq. (34). Suppose that individual errors generate $\epsilon_{i}$, where $i=1,2, \cdots, N$ and their multiplied and combined PSSN are $\operatorname{PSSN}_{M}$ and $\operatorname{PSSN}_{C}$, respectively. Then, the difference, $\Delta_{T}$, between multiplied and combined PSSN becomes

$$
\begin{aligned}
\Delta_{T}= & \sum_{i=1}^{N} \sum_{j=i+1}^{N}\left(\left\langle\epsilon_{i} \epsilon_{j}\right\rangle-\left\langle\epsilon_{i}\right\rangle\left\langle\epsilon_{j}\right\rangle\right) \\
& -\sum_{i=1}^{N} \sum_{j=i+1}^{N} \sum_{k=j+1}^{N}\left(\left\langle\epsilon_{i} \epsilon_{j} \epsilon_{k}\right\rangle-\left\langle\epsilon_{i}\right\rangle\left\langle\epsilon_{j}\right\rangle\left\langle\epsilon_{k}\right\rangle\right) \\
& +\cdots+(-1)^{N}\left(\left\langle\epsilon_{1} \epsilon_{2} \cdots \epsilon_{N}\right\rangle-\left\langle\epsilon_{i}\right\rangle\left\langle\epsilon_{j}\right\rangle \cdots\left\langle\epsilon_{N}\right\rangle\right) .
\end{aligned}
$$

We apply the same assumptions to get an approximated boundary $\Delta_{A}$ of $\Delta_{T}$ using the Taylor expansion again, detailed in Appendix $\underline{A}$ :

$$
\begin{aligned}
\Delta_{A} \approx & \frac{\left\langle r^{4}\right\rangle-\left\langle r^{2}\right\rangle^{2}}{\left\langle r^{2}\right\rangle^{2}} \sum_{i=1}^{N} \sum_{j=i+1}^{N}\left(1-\operatorname{PSSN}_{i}\right)\left(1-\mathrm{PSSN}_{j}\right) \\
& -\frac{\left\langle r^{6}\right\rangle-\left\langle r^{2}\right\rangle^{3}}{\left\langle r^{2}\right\rangle^{3}} \sum_{i=1}^{N} \sum_{j=i+1}^{N} \sum_{k=j+1}^{N}\left(1-\mathrm{PSSN}_{i}\right) \\
& \times\left(1-\mathrm{PSSN}_{j}\right)\left(1-\mathrm{PSSN}_{k}\right)+\cdots+(-1)^{N} \\
& \times \frac{\left\langle r^{2 N}\right\rangle-\left\langle r^{2}\right\rangle^{N}}{\left\langle r^{2}\right\rangle^{N}} \prod_{i=1}^{N}\left(1-\mathrm{PSSN}_{i}\right),
\end{aligned}
$$

where $\operatorname{PSSN}_{i}$ is the PSSN value for the $i$ th error. We further assume that the errors have the same PSSN (uniformly distributed errors in PSSN value sense); then we approximate $\Delta_{A}$ as in Eq. (표): 


$$
\begin{aligned}
\Delta_{A} & \approx \sum_{n=2}^{N}(-1)^{n} \frac{\left\langle r^{2 n}\right\rangle-\left\langle r^{2}\right\rangle^{n}}{\left\langle r^{2}\right\rangle^{n}} \\
& \times\left(1-N \sqrt{1-\mathrm{PSSN}_{\text {multiplied }}}\right)^{n} .
\end{aligned}
$$

Figure 2 shows the analytically calculated $\Delta_{A}$ using Eq. (38) as a function of the number of errors $(N)$ when the multiplied PSSNs are $0.8,0.85$, and 0.9 , respectively. When the multiplied PSSN is 0.85 , the $\Delta_{A}$ is near $7 \times 10^{-3}$ and increases to around $2.1 \times 10^{-2}$ as the number of errors increases from 2 to 100 . This implies that the multiplicative feature is valid within $2 \%$ when the science loss of all combined errors is smaller than $15 \%$.

\section{Relation to Wave Front Errors}

Using Eqs. (16), (19), and (21), we expand the PSSN in terms of $\overline{\Omega:}$

$$
\operatorname{PSSN}=\left\langle\sum_{p=0}^{\infty} \sum_{n=0}^{p} \frac{(-j)^{p}}{n !(p-n) !}\left\langle\Omega^{n}\right\rangle_{r}\left\langle\Omega^{p-n}\right\rangle_{r}\right\rangle .
$$

If we normalize the OPD such that $k \mathrm{OPD}(\vec{r})=\sigma S(\vec{r})$, where $\sigma$ represents the RMS WFE in radians and $S(\vec{r})$ is the normalized OPD in unit-less dimension, i.e., $\int A(\vec{r}) S(\vec{r})^{2} \mathrm{~d} \vec{r}=1$, then $\Omega$ can be represented with $\sigma$ :

$$
\Omega(\vec{r}, \lambda \vec{f})=\sigma \Delta S(\vec{r}, \lambda \vec{f}),
$$

where $\Delta S(\vec{r}, \lambda \vec{f})$ is defined as $S(\vec{r})-S(\vec{r}-\lambda \vec{f})$. Then, Eq. (39) can be represented as

$$
\mathrm{PSSN}=1+a_{1} \sigma+a_{2} \sigma^{2}+\cdots,
$$

where the $a_{i}$ coefficients are defined as

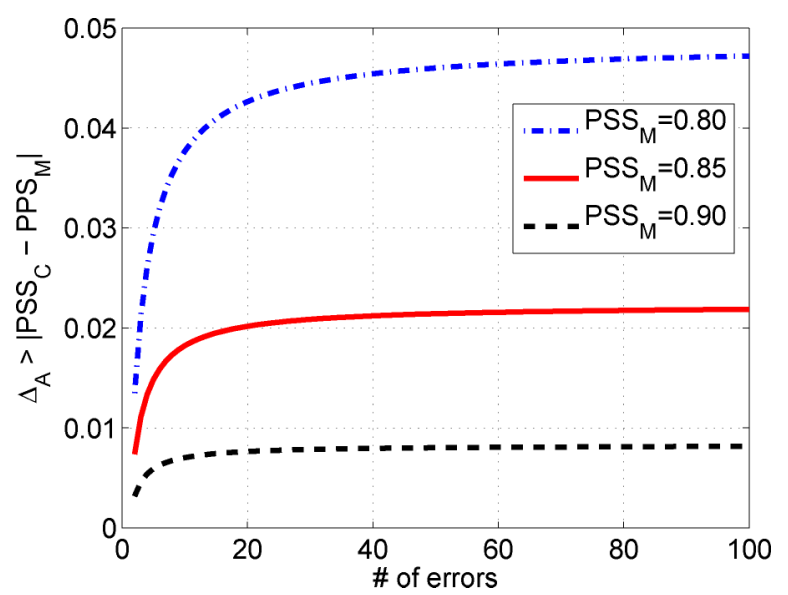

Fig. 2. (Color online) Analytically calculated $\Delta_{A}$ using Eq. (38) as a function of number of errors $(N)$ when the multiplied PSSNs are $0.8,0.85$, and 0.9 , respectively. $\Delta_{A}$ represents the maximum boundary for difference between combined and multiplied PSSN. We assume $A 1$ to $A 6$ are all valid. We also assume that errors have the same PSSN (uniformly distributed errors in PSSN value sense).

$$
a_{p}=\sum_{n=0}^{p} \frac{(-j)^{p}}{n !(p-n) !}\left\langle\left\langle\Delta S^{n}\right\rangle_{r}\left\langle\Delta S^{p-n}\right\rangle_{r}\right\rangle .
$$

Note that the odd coefficients are all pure imaginary numbers, while the even coefficients are all real, suggesting that the odd orders vanish since the OTF absolute square is a real number. Therefore, Eq. (41) shows that the first dominant term with respect to RMS WFE is second order and the PSSN is an even function to RMS WFE. This proves $P 3$ in Section $\underline{3}$.

\section{Relation to Strehl Ratio}

We find that the $\alpha$ value in $P 3$ is the negative value of $a_{2}$ in Eq. (느); i.e.,

$$
\alpha=-a_{2}=\left\langle\langle\Delta S(\vec{r}, \lambda \vec{f})\rangle_{r}^{2}+\left\langle\Delta S(\vec{r}, \lambda \vec{f})^{2}\right\rangle_{r}\right\rangle .
$$

Suppose that the aberration is statistically random (within atmosphere exposure time), the OPD of the aberration is zero mean, and the correlation length of the aberration is zero (i.e., zero-mean white noise). If $E[\cdot]$ denotes the time average (or statistic average) of the aberration, then $\alpha$ becomes

$$
\alpha=\left\langle\langle E[\Delta S(\vec{r}, \lambda \vec{f})]\rangle_{r}^{2}+\left\langle E\left[\Delta S(\vec{r}, \lambda \vec{f})^{2}\right]\right\rangle_{r}\right\rangle .
$$

Since $E[\Delta S(\vec{r}, \lambda \vec{f})]$ is 0 and

$$
\begin{aligned}
E\left[\Delta S(\vec{r}, \lambda \vec{f})^{2}\right]= & E\left[(S(\vec{r})-S(\vec{r}-\lambda \vec{f}))^{2}\right] \\
= & \left(E\left[S(\vec{r})^{2}\right]+E\left[S(\vec{r}-\lambda \vec{f})^{2}\right]\right. \\
& -2 E[S(\vec{r}) S(\vec{r}-\lambda \vec{f})])=2,
\end{aligned}
$$

we find the $\alpha$ value for the white noise aberration:

$$
\alpha=2 .
$$

This implies that the energy spreads out beyond any $r_{o}$ if the aberration is white noise and the PSSN becomes the square of the Strehl ratio. This proves $P 4$ in Section $\underline{3}$.

\section{E. Relation to Central Intensity Ratio}

The CIR is similar to the Strehl ratio but includes the atmosphere; i.e., it is an intensity-based ratio, defined as the central intensity given by the aberrated telescope divided by the central intensity given by an equivalent perfect telescope [4] . Therefore, the CIR can be denoted as

$$
\mathrm{CIR}=\frac{\operatorname{PSF}_{t+a+e}(\vec{\theta}=0)}{\operatorname{PSF}_{t+a}(\vec{\theta}=0)}
$$

Since the OTF and the PSF have a Fourier transform relation, Eq. (47) can be represented using OTFs:

$$
\mathrm{CIR}=\frac{\int_{\infty} \mathrm{OTF}_{t+a+e}(\vec{f}) \mathrm{d} \vec{f}}{\int_{\infty} \mathrm{OTF}_{t+a}(\vec{f}) \mathrm{d} \vec{f}}
$$


We apply the same technique as Eq. (19) to denote the CIR as

$$
\mathrm{CIR}=\left\langle\mathrm{OTF}_{e}(\vec{f})\right\rangle_{\mathrm{CIR}}
$$

where $\langle\cdot\rangle_{\mathrm{CIR}}$ defines a new operator, average over the atmosphere in CIR space, such that

$$
\langle\cdot\rangle_{\mathrm{CIR}} \equiv \frac{\int_{\infty}(\cdot) \mathrm{OTF}_{t+a}(\vec{f}) \mathrm{d} \vec{f}}{\int_{\infty} \mathrm{OTF}_{t+a}(\vec{f}) \mathrm{d} \vec{f}}
$$

Thus, mathematically, the CIR is the average of $\mathrm{OTF}_{e}$ over $\mathrm{OTF}_{t+a}$. We also expand the CIR using its Taylor expansion with respect to the RMS WFE $\sigma$ :

$$
\mathrm{CIR}=1+b_{1} \sigma+b_{2} \sigma^{2}+\cdots
$$

where the $b_{i}$ coefficients are defined as

$$
b_{n}=\frac{(-j)^{n}}{n !}\left\langle\left\langle\Delta S(\vec{r}, \lambda \vec{f})^{n}\right\rangle_{\vec{r}}\right\rangle_{\mathrm{CIR}}
$$

Note also that odd coefficients are all pure imaginary numbers while even coefficients are all real, suggesting that odd orders are all vanishing since the CIR is a real number. Therefore, Eq. (51) shows that the first dominant term with respect to RMS WFE is the second order and the CIR is an even function to RMS WFE. The CIR can be approximated up to the second order of the RMS WFE if we assume the RMS WFE is small:

$$
\mathrm{CIR} \approx 1-\beta \sigma^{2}
$$

where $\beta$ is also a proportional constant independent to $\sigma$ for CIR. Therefore, together with $P 3$, the PSSN has a relation to CIR as in P5, where $\gamma$ is defined as

$$
\gamma \equiv \frac{\alpha}{\beta}
$$

In addition, if we suppose that the OPD of the aberration is zero mean and the correlation length of the aberration is zero (i.e., zero-mean white noise), one can find that the $\beta$ value becomes 1 using Eq. (45). This implies that the energy spreads out beyond any $r_{o}$ if the aberration is white noise and the CIR becomes the Strehl ratio. Therefore, we prove P5 in Section 3.

However, we observe that the second order approximation made in Eq. (53) fails for certain aberrations such as Zernike tip/tilt or coma aberrations. For those aberrations, a higher order term than the second order becomes dominant. It is due to this that we define the CIR at the PSF center of the unaberrated telescope.
F. Spatial Frequency Dependence of Normalized Point Source Sensitivity

In order to demonstrate the spatial frequency dependence of PSSN, we calculate the PSSN using the following analytical model:

M1 We assume an infinitely large telescope, so the OTF is only limited by the atmosphere and/or the telescope aberrations; i.e., $\mathrm{OTF}_{t+a+e}(\vec{f})=\mathrm{OTF}_{a+e}(\vec{f})$ and $\mathrm{OTF}_{t+a}(\vec{f})=\mathrm{OTF}_{a}(\vec{f})$.

M2 We assume long exposure Kolmogorov atmosphere [8] with Fried parameter of $r_{o}$ for the atmospheric aberration. Then, $\mathrm{OTF}_{a}(\vec{f})$ can be defined as Eq. (30).

M3 The telescope aberration is random shiftinvariant and isotropic. Its structure function has the form of

$$
D(r)=\sigma_{\phi}^{2}\left(1-\exp \left(-\left(\frac{r}{\sigma_{d}}\right)^{2}\right)\right)
$$

where $\sigma_{\phi}$ is the RMS WFE in unit of radians and $\sigma_{d}$ is the correlation length of the aberration in unit of meters.

We calculated the PSSN analytically using this model by varying parameters of $r_{o}, \sigma_{\phi}$, and $\sigma_{d}$. The $x$ and $y$ axes in Fig. 3 show the Fried parameter normalized to correlation length of the aberration $\left(r_{o} / \sigma_{d}\right)$ and calculated $\alpha_{o}=(1-\mathrm{PSSN}) / \sigma_{\phi}^{2}$ values, respectively. Note that $\alpha_{o}$ is a sum of the $\alpha$ value and higher order residuals as defined in $P 3$ and Subsection 4.C $\left(\alpha_{o}=(1-\mathrm{PSSN}) / \sigma_{\phi}^{2}=\alpha+a_{4} \sigma_{\phi}^{2}+a_{6} \sigma_{\phi}^{4}+\cdots\right)$. Therefore, $\alpha_{o}$ approaches $\alpha$ as $\sigma_{\phi}$ decreases.

Figure 3 illuminates a few key characteristics of PSSN. First, as the correlation length of the aberration decreases (and its relative spatial frequency $r_{o} / \sigma_{d}$ increases), the aberration becomes stronger compared to the atmospheric aberrations. This in turn results in decreasing PSSN (increasing $\alpha_{o}$ ) even

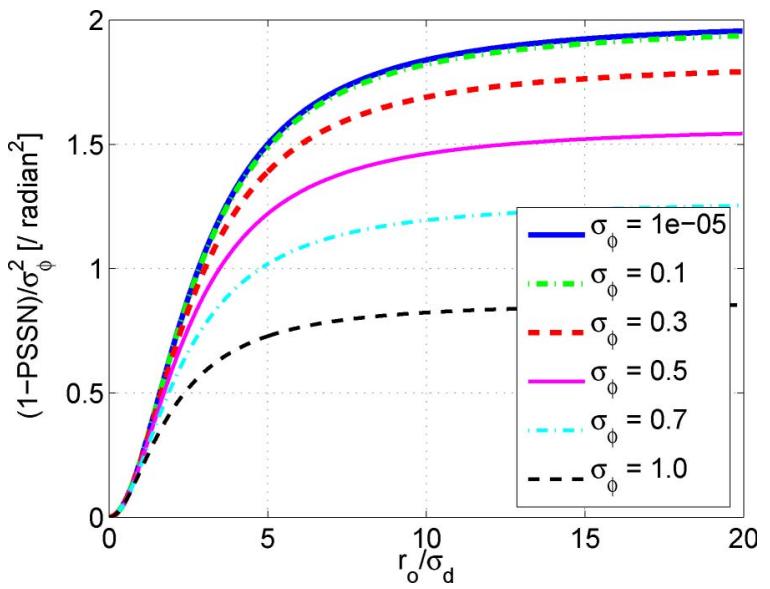

Fig. 3. (Color online) Analytically calculated PSSN using our simple analytical model (M1 to $M 3$ ). The $x$ and $y$ axes show the Fried parameter normalized to correlation length of the aberration $\left(r_{o} / \sigma_{d}\right)$ and calculated $\alpha_{o}=(1-\mathrm{PSSN}) / \sigma_{\phi}^{2}$ values, respectively. 
for the same RMS WFE. We can also observe that $\alpha_{o}$ asymptotically approaches $2 / \mathrm{rad}^{2}$ for very small $\sigma_{\phi}$, just as $P 4$ suggests.

Second, $\alpha_{o}$ becomes independent of $\sigma_{\phi}$ as $\sigma_{\phi}$ becomes small. This again illustrates the validity of $P 3$ if RMS WFE $\left(\sigma_{\phi}\right)$ is small.

Third, in the most practical regime when aberration frequency is much lower than the atmospheric frequency $\left(r_{o} / \sigma_{d} \ll 1\right), \alpha_{o}$ converges to the low frequency $\alpha$ values shown in Table 1 . More importantly, Fig. 3 also indicates that $\alpha_{o}$ is virtually independent of RMS WFE in a significant frequency range for low frequencies. In other words, Eq. (6) is valid up to relatively high spatial frequency errors.

\section{Numerical Analysis}

\section{A. Simulation Method}

In order to verify the properties of the PSSN that we have investigated in the analytical studies in the Section $\underline{4}$, we perform numerical simulations with aberrations on a $30 \mathrm{~m}$ circular pupil. Figure 4 shows the numerical calculation procedure for the PSSN with an example of Zernike mode 800 aberration (all Zernike modes in this paper refer to Zernike Noll modes [9]). First, we introduce Zernike aberrations in the pupil map. After we obtain their PSFs and OTFs using the fast Fourier transform (FFT) algorithm built into Matlab (The MathWorks, Inc.), we produce the atmosphere-exposed OTFs by multiplying the OTFs and long-exposure atmosphere OTF [8] denoted in Eq. (30). Then, we obtain the $\mathrm{PSS}_{t+a+e}[\mathrm{be}-$ fore normalization as in the numerator in Eq. (6)] in the OTF domain using the Parseval's energy conservation theorem. This computation flow is shown across the top of Fig. 4 . Likewise, across the bottom of Fig. 4, we compute the atmosphere-exposed PSS for ideal telescope [before normalization as in the denominator in Eq. (6)] using an aberrated OPD map. Finally, we obtain the normalized PSS by dividing $\operatorname{PSS}_{t+a+e}$ by $\operatorname{PSS}_{t+a}$.
We use a $1 / 64 \mathrm{~m}$ per pixel sampling grid with $2048 \times 2048$ pixels across the pupil, a Nyquist sampling for FFT, a Fried parameter $r_{o}$ of $200 \mathrm{~mm}$, and a wavelength of $500 \mathrm{~nm}$ for the numerical simulation parameters.

We also obtain the CIR values using a similar calculation procedure. Calculating CIR values differs from our method of calculating PSSN values by integration of the OTF (CIR) versus the OTF squared (PSSN) in the numerator and denominator as shown in Fig. 4.

\section{B. Simulation Results}

For Zernike aberrations of mode 4, 100, 200, 400, and 800 , we compute the PSSN with respect to its RMS WFE, and the results are shown in Fig. 5(a). The simulated RMS WFE ranges from 0 to $160 \mathrm{~nm}$. For all aberrations, the PSSN is 1 when the RMS WFE is zero and decreases as the RMS WFE increases. The PSSN decreases faster for higher-mode Zernike aberrations, as expected. A log-log scale plot of $1-$ PSSN is shown in Fig. 5(b). Note that the plots in Fig. 5(b) are close to linear lines if the RMS WFE is small and the ratio between the $x$ and $y$ axes is 2 , verifying $P 3$. The linear lines in Fig. 5(b) begin to distort as the RMS WFE increases. Higher-order Zernikes distort earlier than lower-order Zernikes.

We have performed similar simulations for other Zernikes and computed the $\alpha$ values in Eq. (9). Table 1 summarizes the results in the fifth column. The $\alpha$ values are numerically evaluated at the RMS WFE of $2 \mathrm{~nm}$. From the results, we observe that the $\alpha$ values of lower azimuthal aberrations are larger in the same radial Zernike order. This is because the atmosphere we consider is isotropic; thus, lower azimuthal (or higher radial) aberrations become more sensitive (lower PSSN values) than higher azimuthal (or less radial) aberrations to the point source. We also find that the $\alpha$ value for the white noise is close to 2, as we expect in $P 4$ in Eq. (10).

Table 1. Numerically Calculated $\alpha, \beta$, and $\gamma$ Values

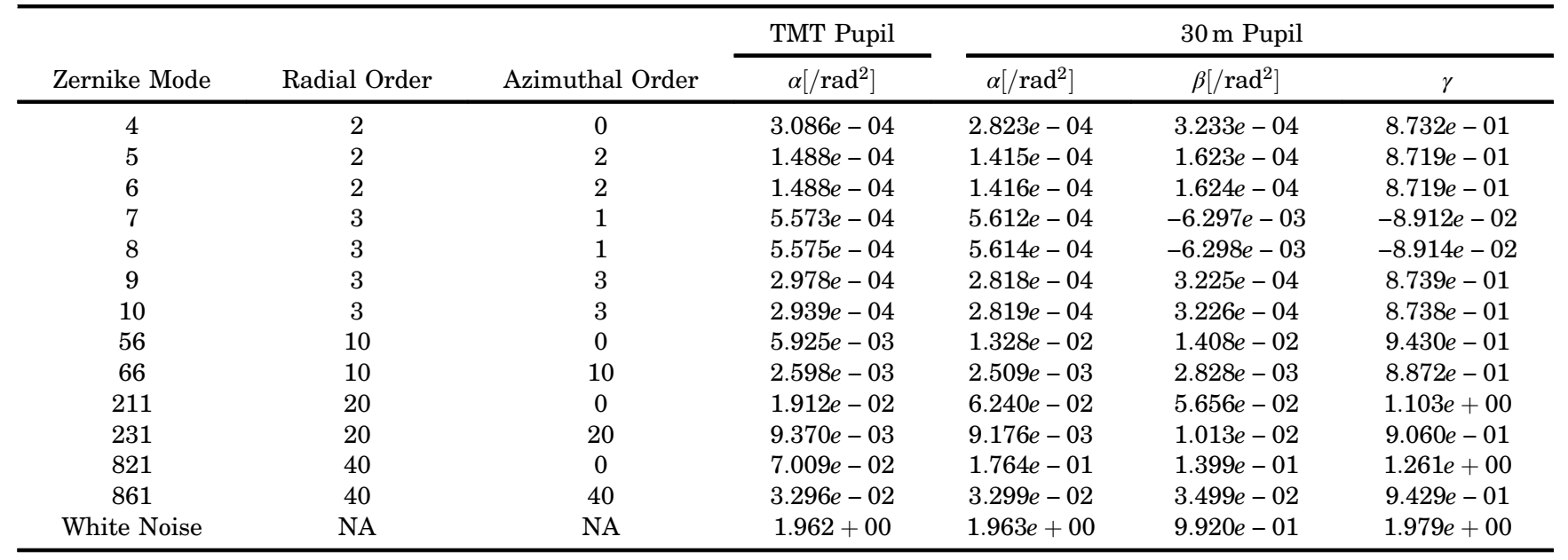

${ }^{a}$ Its graphical representation is shown in Fig. $\underline{6}$. 


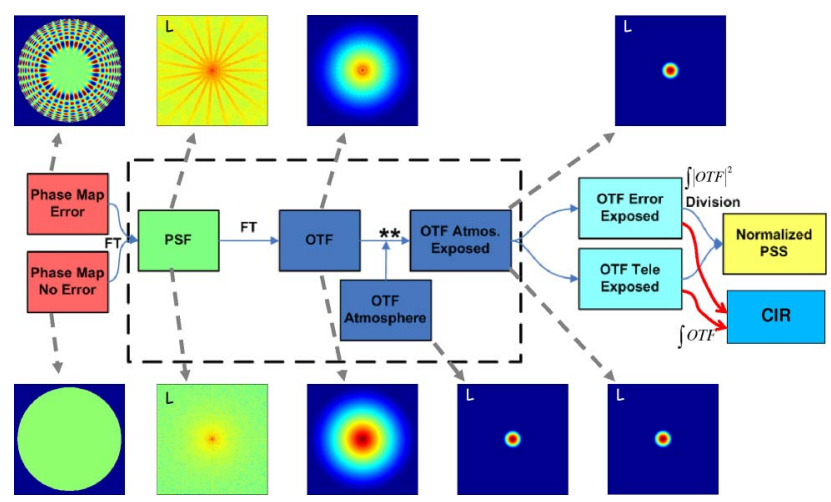

Fig. 4. (Color online) Numerical procedure to calculate PSSN with Zernike mode 800 aberration for example. The "L" in top left on the figures denotes log scale plot.

The fourth column of Table 1 shows $\alpha$ values calculated using the TMT pupil. The TMT pupil we consider includes 492 hexagonal segments with segment gaps, the secondary mirror obscuration, and no support obscuration [3]. The $\alpha$ values for the two pupil maps are similar except that the $\alpha$ values for the TMT pupil become smaller than those of the ideal circular pupil when the azimuthal mode is small. This is because the circular pupil includes more outside region than the TMT pupil, which contains more higher frequency aberrations.

Using the same aberrations, we also computed CIR values to compare with PSSN. Figure 6(a) shows numerically calculated $\alpha$ and $\beta$ values for Zernike modes, and Fig. $6(\mathrm{~b})$ shows their ratios $(\gamma=\alpha / \beta)$. The $\beta$ and $\gamma$ values are also listed in the sixth and seventh columns, respectively, in Table 1 . We observe the following relations between PSSN and CIR. First, PSSN and CIR are well correlated for lowerorder Zernikes aberrations simulated as seen in Fig. 6(a). The two metrics become more similar when the radial order is smaller and the azimuthal order is larger. Second, we observe PSSN is larger than CIR $(\gamma<1)$ for smaller Zernike modes and smaller $(\gamma>1)$ for higher Zernike modes. The $\gamma$ values are near 0.87

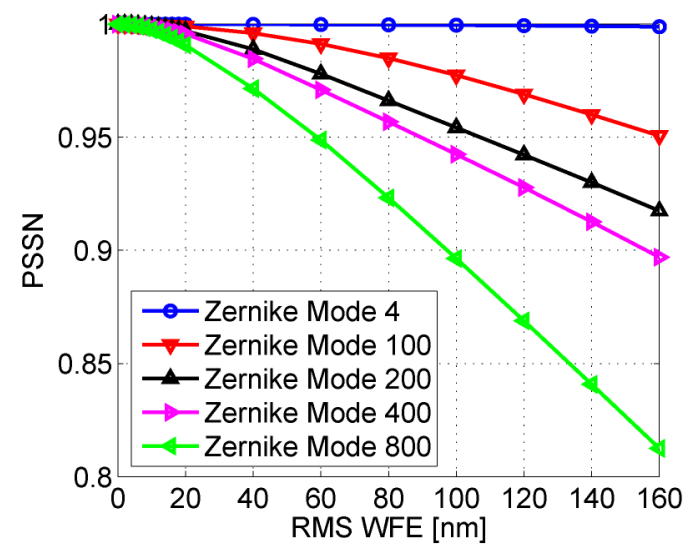

(a) PSSN vs RMS for Zernike modes smaller than 10 and increase as the Zernike mode increases. As discussed in P5, the maximum limits for $\alpha$ and $\beta$ are 2 and 1, respectively, when the aberration is the white noise. Therefore, the $\gamma$ value is expected to approach 2 as the Zernike mode becomes larger. The $\gamma$ value for the white noise in Table 1 also demonstrates this.

In order to verify the multiplicative feature of PSSN (P2), we first obtain combined OPDs by adding all OPDs that have equal RMS WFEs for the Zernikes shown in Fig. 5 and then calculate the combined PSSNs and RMS WFEs for the combined OPDs. We also obtain multiplied PSSNs by multiplying the PSSN values for all considered Zernikes using the same RMS WFE. Then, we compare the combined PSSNs to multiplied PSSNs. Figure 7 shows the simulation results. The difference between combined and multiplied PSSN values is small when the RMS WFE is small (or the multiplied PSSN is close to 1) and increases as the RMS WFE increases (or the multiplied PSSN becomes worse). The $x$ axis in Fig. 7 represents the computed RMS WFE for the combined OPDs, which are same as the RSSed RMS WFE due to the orthogonality of Zernikes.

Note that Zernikes are nonindependent errors (see A3), while our analytical boundary of $\Delta_{A}$ in Eq. (37) is approximated for independent errors. Therefore, the numerical $\Delta_{N}$ cannot be directly compared to the analytical boundary $\Delta_{A}$. For a more quantitative verification of the analytical boundary $\Delta_{A}$, we use the TMT primary errors in comparing numerical $\Delta_{N}$ and analytical boundary $\Delta_{A}$; Fig. 8 shows the result. The TMT primary errors include segment figuring residual, segment thermal distortion, segment support print-through, segment in-plane residual, $M 1$ segment out-of-plane residual, and $M 1$ segment dynamic displacement residual. We have described these errors in detail in our previous studies[3].

Since the TMT $M 1$ primary errors include random errors that are potentially independent of each other, discussed in $A 3$ (however, we have not verified whether they are independent or not), $\Delta_{A}$ is expected

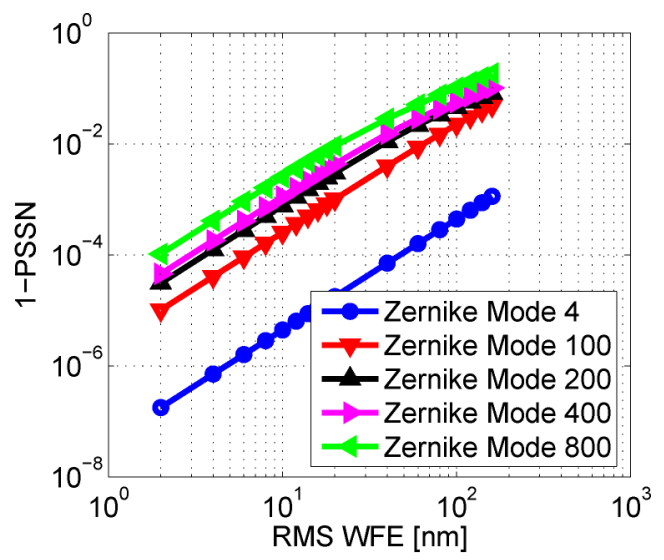

(b) 1-PSSN vs RMS (log-log)

Fig. 5. (Color online) Numerically calculated PSSN for Zernike 4, 100, 200, 400, and 800 modes with respect to the RMS WFE: (a) PSSN plot in linear scale and (b) 1 - PSSN plot in a log-log scale. 


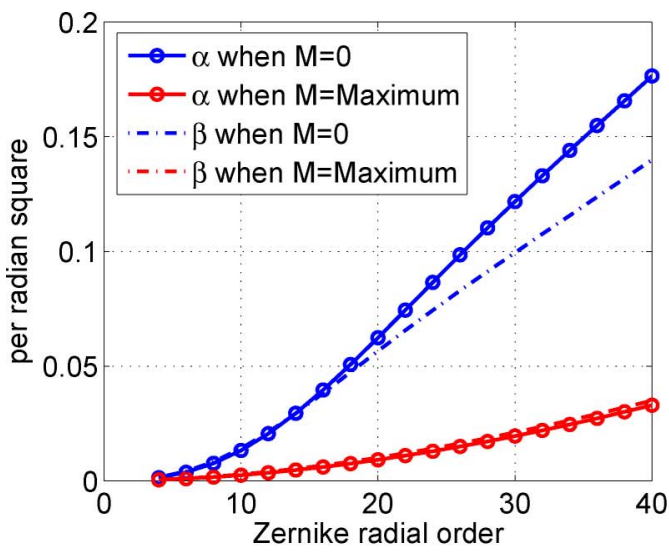

(a) $\alpha$ and $\beta$ vs Zernike radial order

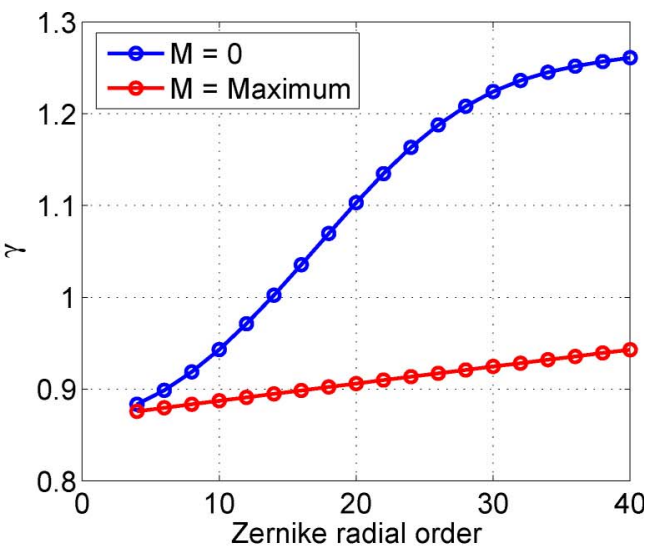

(b) $\gamma$ vs Zernike radial order

Fig. 6. (Color online) Numerically calculated (a) $\alpha, \beta$, and (b) $\gamma$ values for various Zernike modes. The azimuthal index, $M$, for the simulated Zernike modes are either maximum or zero mode for a given Zernike radial index [].

to estimate the boundary of $\Delta_{N}$ better than in Zernike aberrations. As seen in Fig. 8 , the numerically obtained $\Delta_{N}$ values are within the analytical boundary of $\Delta_{A}$ for most zenith angles, supporting that our analytical studies and approximations are valid for the practical errors within the TMT.

\section{Summary}

Among other science metrics, the normalized PSS is distinguished in that it includes atmosphere information by appropriately weighting telescope errors in terms of atmosphere. Therefore, we believe that the normalized PSS is truly a measure of the science loss for seeing-limited instruments. Furthermore, it has a multiplicative feature when combining multiple error sources. In this paper, we find that the multiplicative feature is valid within a few percentages (within $2 \%$ for independent errors) when the science loss of all combined errors is smaller than $15 \%$ (i.e., the combined PSSN is larger than $85 \%$ ). Since TMT

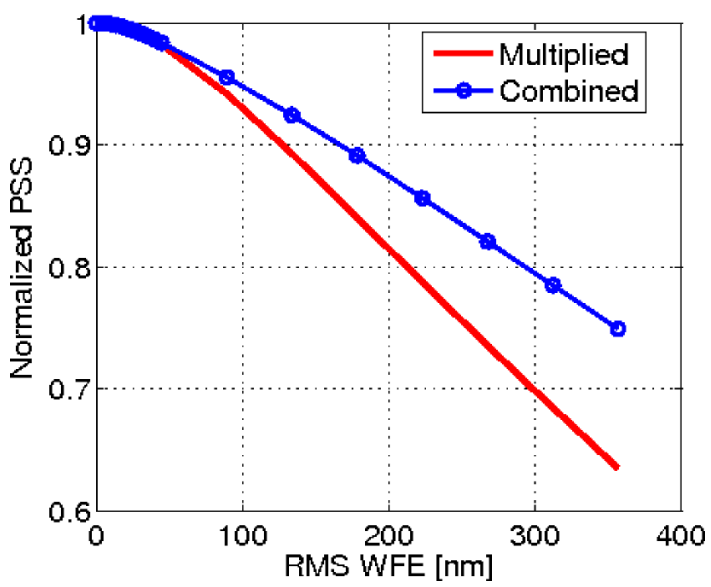

Fig. 7. (Color online) Combined and multiplied PSSN. We first obtain combined OPDs by adding all OPDs that have equal RMS WFEs for the Zernikes shown in Fig. 5 and then calculate the combined PSSNs and RMS WFEs for the combined OPDs. We also obtain multiplied PSSNs by multiplying the PSSN values for all considered Zernikes using the same RMS WFE. has a requirement that the telescope should not introduce more than a $20 \%$ loss of science (the goal of the TMT that seeing-limited performance of the telescope achieves $80 \%$ of that of a perfect telescope is in terms of "science performance ratio," and its corresponding goal in terms of PSSN is around 0.85 $[1,2])$, we believe that this metric is useful for optical designing and budgeting errors of large, groundbased telescopes such as TMT.

\section{Proof of Eq. (33)}

Suppose that the assumptions (from $A 1$ to $A 6$ ) made in Subsection 4.B are all valid and two different errors generate $\overline{\epsilon_{1}}$ and $\epsilon_{2}$, respectively. If their PSSN values are $\mathrm{PSS}_{1}$ and $\mathrm{PSS}_{2}$, respectively, then we prove the following is true:

$$
\Delta \equiv\left\langle\epsilon_{1} \epsilon_{2}\right\rangle-\left\langle\epsilon_{1}\right\rangle\left\langle\epsilon_{2}\right\rangle \approx 1.2119\left(1-\mathrm{PSS}_{1}\right)\left(1-\mathrm{PSS}_{2}\right) .
$$

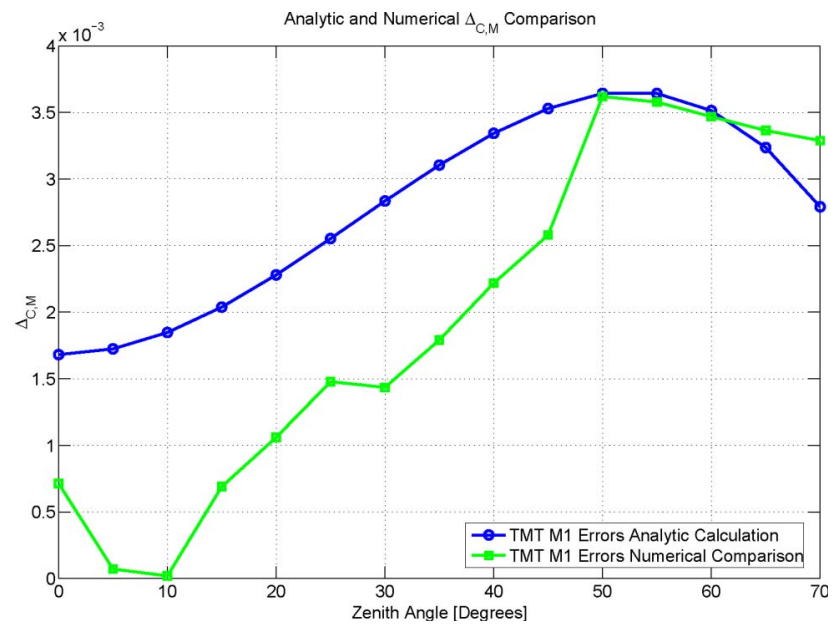

Fig. 8. (Color online) Numerical difference $\Delta_{N}$ and analytical difference boundary $\Delta_{A}$ between combined and multiplied PSSN for the TMT M1 primary errors. The two curves are obtained using two independent methods. Numerical $\Delta_{N}$ values are from studies done by Nissly, et al. [3], and the analytical boundary $\Delta_{A}$ is from Eq. (37). 
[Proof.]

First, we approximate individual $\epsilon_{i}(x, y)$ as

$$
\epsilon_{i}(x, y)=a_{1} x+b_{1} y+a_{2} x^{2}+b_{2} x^{2}+c_{1,1} x y+\ldots,
$$

where the coefficients, $a_{k}, b_{k}$, and $c_{i, j}$ are

$$
\begin{gathered}
\left.\frac{1}{k !} \frac{\partial^{k} \epsilon}{\partial x^{k}}\right|_{(0,0)}, \\
\left.\frac{1}{k !} \frac{\partial^{k} \epsilon}{\partial y^{k}}\right|_{(0,0)}, \\
\left.\frac{{ }_{i+j} C_{i}}{(i+j) !} \frac{\partial^{i+j_{\epsilon}}}{\partial^{i} x \partial^{i_{y}}}\right|_{(0,0)},
\end{gathered}
$$

respectively. Since the OTF has a Fourier transformation relation to the PSF and the PSF is a real positive valued function, according to the Fourier transform property, OTFs (hence $\epsilon$ ) are even functions. Therefore, the odd orders are all dropped in Eq. (57). With the further assumption that these errors are isotropic in the $\vec{f}$ domain (i.e., $a_{k}=b_{k}=\alpha_{k}$ and $\left.c_{i, j}=0\right)$ and if we approximate Eq. (57) up to second order terms (A2), then

$$
\epsilon_{i}(r) \approx \alpha_{2} r^{2} .
$$

Therefore, $\Delta$ can be approximated as

$$
\Delta=p_{2} q_{2}\left(\left\langle r^{4}\right\rangle-\left\langle r^{2}\right\rangle^{2}\right),
$$

where $p_{2}$ and $q_{2}$ are $\alpha_{2}$ for $\epsilon_{1}$ and $\epsilon_{2}$, respectively. Since we know the individual PSSN values,

$$
\begin{aligned}
& 1-\mathrm{PSS}_{1}=\left\langle\epsilon_{1}\right\rangle=p_{2}\left\langle r^{2}\right\rangle, \\
& 1-\mathrm{PSS}_{2}=\left\langle\epsilon_{2}\right\rangle=q_{2}\left\langle r^{2}\right\rangle,
\end{aligned}
$$

Eq. (59) becomes

$$
\Delta \approx \frac{\left.\left(\left\langle r^{4}\right\rangle\right)-\left\langle r^{2}\right\rangle^{2}\right)}{\left\langle r^{2}\right\rangle^{2}}\left(1-\mathrm{PSS}_{1}\right)\left(1-\mathrm{PSS}_{2}\right)
$$

Once the atmosphere OTF is defined as in $A 6$,

$$
\frac{\left(\left\langle r^{2}\right\rangle-\left\langle r^{2}\right\rangle^{2}\right)}{\left\langle r^{2}\right\rangle^{2}}
$$

is a scalar value that can be computed to a finite value. We find the constant as $1.2119 .$. ; therefore, we prove Eq. (56).

This research was carried out in part at the Jet Propulsion Laboratory, California Institute of Technology, and was sponsored by the California Institute of Technology and the National Aeronautics and Space Administration (NASA). The authors gratefully acknowledge the support of the TMT partner institutions. They are the Association of Canadian Universities for Research in Astronomy (ACURA), the California Institute of Technology, and the University of California. This work was supported as well by the Gordon and Betty Moore Foundation, the Canada Foundation for Innovation, the Ontario Ministry of Research and Innovation, the National Research Council of Canada, the Natural Sciences and Engineering Research Council of Canada (NSERC), the British Columbia Knowledge Development Fund, the Association of Universities for Research in Astronomy (AURA), and the U.S. National Science Foundation (NSF).

\section{References}

1. G. Z. Angeli, S. Roberts, and K. Vogiatzis, "Systems engineering for the preliminary design of the Thirty Meter Telescope," Proc. SPIE 7017, 701704 (2008).

2. K. Vogiatzis and G. Z. Angeli, "Monte Carlo simulation framework for TMT," Proc. SPIE 7017, 7017V (2008).

3. C. Nissly, B. Seo, M. Troy, G. Angeli, J. Angione, I. Crossfield, B. Ellerbroek, L. Gilles, and N. Sigrist, "High-resolution optical modeling of the Thirty Meter Telescope for systematic performance trades," Proc. SPIE 7017, 70170U (2008).

4. P. Dierickx, "Optical performance of large ground-based telescopes," J. Mod. Opt. 39, 569-588 (1992).

5. I. R. King, "Accuracy of measurement of star images on a pixel array," Publ. Astron. Soc. Pac. 95, 163-168 (1983).

6. R. A. Muller and A. Buffington, "Real-time correction of atmospherically degraded telescope images through image sharpening," J. Opt. Soc. Am. 64, 1200-1210 (1974).

7. J. C. Christou and R. B. Makidon, "Strehl ratio and image sharpness for adaptive optics," Proc. SPIE 6272, 62721Y (2006).

8. J. Hardy, Adaptive Optics for Astronomical Telescopes (Oxford Univ. Press, 1998).

9. R. J. Noll, "Zernike polynomials and atmospheric turbulence," J. Opt. Soc. Am. 66, 207-211 (1976). 